\title{
The relationship between inflammatory bowel disease and Helicobacter pylori across East Asian, European and Mediterranean countries: a meta-analysis
}

\author{
Rabbiaatul Addawiyah Imawana, Daniel Robert Smith, Michaela Louise Goodson
}

Newcastle University Medicine Malaysia

Abstract

Background The current literature suggests a protective benefit of Helicobacter pylori (H. pylori) infection against inflammatory bowel disease (IBD). Here we assessed whether this effect varied by IBD subtype-Crohn's disease (CD) or ulcerative colitis (UC) - and geographic region: East Asia, Europe (non-Mediterranean) or Mediterranean region.

Methods A database search was performed up to July 2019 inclusive for all studies that compared H. pylori infection in IBD patients vs. non-IBD controls. The relative risk (RR) was used to quantify the association between IBD and $H$. pylori, and the effects were combined across studies using a mixed-effects meta-regression model, which included IBD subtype and geographic region as categorical moderator variables.

Results Our meta-regression model exhibited moderate heterogeneity $\left(\mathrm{I}^{2}=48.74 \%\right)$. Pooled RR depended on both region $(\mathrm{P}=0.02)$ and subtype $(\mathrm{P}<0.001)$. Pooled RRs were $<1$ for all subtype and region combinations, indicative of a protective effect of $\mathrm{H}$. pylori against IBD. The pooled RR was $28 \%(9 \%, 50 \% ; \mathrm{P}=0.001)$ greater for $\mathrm{UC}$ vs. $\mathrm{CD}$ and $43 \%(4 \%, 96 \% ; \mathrm{P}=0.02)$ greater for Mediterranean countries vs. East Asia. The pooled RR was $18 \%(-13 \%, 60 \% ; \mathrm{P}=0.48)$ greater for Europe vs. East Asia and 21\% (-13\%, 68\%; P=0.42) greater for Mediterranean vs. Europe, though these differences were not statistically significant.

Conclusions The protective effect of $H$. pylori on IBD varied by both subtype (more protection against $C D$ vs. UC) and region (East Asia more protected than Mediterranean regions). Variation due to these effects could provide insight into IBD etiology.

Keywords East Asia, Europe, Mediterranean, Helicobacter pylori, inflammatory bowel disease

Ann Gastroenterol 2020; 33 (4): 1-10

\section{Introduction}

Inflammatory bowel disease (IBD) is a chronic relapsing and remitting inflammation of the gastrointestinal tract, comprising the subtypes Crohn's disease (CD) and ulcerative colitis (UC) [1]. Its global prevalence is estimated to exceed 6 million [2], and in Europe alone more than 2 million people

Medical Research Department, Faculty of Medical Sciences, Newcastle University Medicine Malaysia

Conflict of Interest: None

Correspondence to: Rabbiaatul Addawiyah Imawana, Medical Research Department, Faculty of Medical Sciences, Newcastle University,

Medicine Malaysia (NUMED Malaysia), No. 1, Jalan Sarjana 1, Kota

Ilmu, Malaysia, e-mail: R.A.Binti-Imawana2@newcastle.edu.my

Received 30 January 2020; accepted 6 May 2020;

published online 6 June 2020

DOI: https://doi.org/10.20524/aog.2020.0507 are currently diagnosed with IBD [2-5]. Once considered rare in Asian countries, IBD's incidence has increased considerably in the last 2 decades and prevalence is currently estimated to range between 0.54 and 3.44 per 100,000 persons $[1,4,6,7]$. IBD has become a disease of global significance, not only compromising quality of life, but also leading to complications such as malnutrition and increased risk of gastrointestinal cancer [5,8-11].

Helicobacter pylori (H. pylori) is well known for its unique ability to colonize the acidic environment of the human stomach [12]. Previous studies have reported that the $H$. pylori infection rate is significantly lower in IBD patients compared to non-IBD controls [13-16]. Moreover, there is the suggestion that this protective effect is stronger for CD vs. UC, and in Eastern vs. Western countries [1316]. However, studies of "Eastern" populations have been largely confined to East Asia (China, Japan, Korea), whereas studies of "Western" populations have included a broader range of countries from Europe (e.g., Germany, Italy), North 
America (e.g., Mexico) and South America (e.g., Brazil). Such generalized East vs. West contrasts make it challenging to hypothesize about causal agents [13-16], though certain diets, such as the Mediterranean diet, are purported to be protective of IBD [17-19].

Regional and subtype disparities in the protective effect of $H$. pylori on IBD remain circumstantial and have yet to be formally tested in a meta-analytic framework. Such a study is imperative, since significant effects of region and/or subtype may shed light on IBD's etiology, which is currently unclear [20]. This meta-analysis aims to bridge this important research gap by simultaneously incorporating subtype and region in an all-encompassing meta-regression model.

We studied 3 specific geographic regions, namely East Asia, Europe (non-Mediterranean) and the Mediterranean. The selection of these particular regions was guided by existing studies that recognized differences in potential risk factors, IBD characteristics, or features unique to these populations, such as the Mediterranean diet [6,16,17,21-24]. The publication of a sizeable and approximately balanced number of primary studies from East Asian, European and Mediterranean countries is desirable from a statistical power standpoint and provided further motivation for our study. Our meta-analysis aimed to address the following research questions:

1. Does the association between IBD and H. pylori vary by IBD subtype?

2. Does the association between IBD and H. pylori vary by region?

3. Is there an interaction effect of IBD subtype and region on the association between IBD and H. pylori?

\section{Materials and methods}

This meta-analysis followed the guidelines provided by the Preferred Reporting Items for Systematic Reviews and MetaAnalyses (PRISMA) [25].

\section{Inclusion and exclusion criteria}

Inclusion criteria for studies in our meta-analysis were: 1) studies that examined the association between $H$. pylori and IBD; 2) studies that included adult populations; 3) studies conducted on populations from European, East Asian or Mediterranean countries; 4) studies that reported exact numbers of IBD patients for CD and/or UC subtypes; and 5) studies either originally available in English or could be translated into English. Studies were excluded when they: 1) focused on pediatric populations; 2) focused on countries or regions other than those specified in the above inclusion criteria; and 3) lacked a control group required to compute the effect size (relative risk [RR], see below) to quantify the association between $H$. pylori and IBD.

\section{Search strategy}

A rigorous database search was performed using Scopus, Ovid MEDLINE, Embase, PubMed and Cochrane Library for studies published up until June 2019 inclusive. Our search strategy included Medical Subject Heading (MeSH) terms and keyword combinations such as "Inflammatory Bowel Disease", "IBD”, "Ulcerative Colitis", "UC”, “Crohn's Disease”, “CD”, "H. pylori" and "Helicobacter pylori". Boolean search operators "AND" and "OR" were used to combine search terms. We also performed manual searches using references from studies retrieved for any additional relevant studies.

\section{Data extraction}

Information retrieved from selected studies included titles, authors, publication year, study design (cohort or casecontrol), population age group, population origin or region of the studies, $H$. pylori detection method, and sample sizes in H. pylori (positive/negative) and IBD (case/control) groups. IBD patients were further divided into CD and UC subtypes, along with their results for $H$. pylori infection. Studies were separated into 3 groups, namely East Asian, European (excluding Mediterranean) and Mediterranean, according to the geographic region in which they were conducted.

\section{Risk of bias}

The Newcastle-Ottawa scale was used to assess the risk of bias in individual studies $[26,27]$. Studies were first grouped based on their design, such as case-control or cohort studies, before being assessed for bias. Studies with a score of $\geq 7$ were regarded to be of "higher quality", implying a lower risk of bias [27].

\section{Statistical analysis}

Meta-analysis was performed using $\mathrm{R}$ (predominantly using the metafor and ggplot2 packages), formally known as The R Project for Statistical Computing version 3.6.1 R Core Team (2019) [28-30] (see supplementary material for full reproducible $\mathrm{R}$ code and master dataset). From each primary study, we extracted the counts of positive and negative cases of $H$. pylori in the IBD and non-IBD groups. In line with previous meta-analyses that combined cohort and case-control studies [13-16], we used the RR as our effect size metric to quantify the magnitude of the association between IBD and $H$. pylori incidence. We fitted a hierarchical weighted mixed-effects meta-regression model [31] of the $\log \mathrm{RR}$, which included region (levels: E. Asia; Europe; Mediterranean) and subtype (levels: $\mathrm{CD}$; UC) as categorical (dummy-coded) moderator variables, with the amount of residual heterogeneity estimated using maximum likelihood. To our knowledge, none of our included studies shared the same dataset. However, 19 studies included data on both UC and CD, implying correlated sampling errors. 
Accordingly, we included the study name and observation id as random effects (where observation id was nested in study name) to account for non-independence at the study level. To test the significance of a possible region $\times$ subtype interaction, we performed a likelihood ratio test, comparing the full model (containing region and subtype main effects and the region $x$ subtype interaction term) with a reduced model containing only the additive main effects of region and subtype. We present model predictions (i.e., weighted averages or summary effects) as RRs and corresponding confidence intervals for all moderator combinations. We did not present an overall effect since this is somewhat meaningless and often misleading in the presence of moderators [32]. Contrasts between levels of subtype and region variables (e.g., RR Mediterranean vs. RR E. Asia) were expressed as ratios of RR (RRR) with confidence intervals and $\mathrm{P}$ values adjusted for simultaneous inference using the single-step method. Statistical heterogeneity was assessed using Cochran's Q test (threshold P-value <0.10) and Higgins test $\left(I^{2}\right)$ (low heterogeneity: $I^{2}<25 \%$; moderate heterogeneity: $I^{2}$ 25-75\%; high heterogeneity: $I^{2}>75 \%$ ) $[33,34]$. We performed leave-one-out sensitivity analysis, where the model was iteratively re-fit after omitting each respective study to examine the effect on predictions (RR) and contrasts (RRR). The pseudo Egger regression test was used to assess for small study bias. Here, study variances were included in the selected meta-analysis model as an additional moderator and a P-value was computed to test the null hypothesis that the intercept term was equal to zero (rejection of the null implied evidence of small-study bias). The standard funnel plot was also used to assess for small-study bias and the contour-enhanced funnel plot was used to assess whether any such bias might be attributed to publication bias. With the exception of Cochran's $\mathrm{Q}$ test, we set our significance threshold at $\alpha=0.05$ (i.e., $5 \%$ ), accordingly computing $95 \%$ confidence intervals.

\section{Results}

\section{Search results and main characteristics of studies}

A total of 477 relevant studies were identified through database and manual searches. After screening of the titles and abstracts, 418 irrelevant studies were excluded. The remaining 59 articles were retrieved for detailed evaluation. Upon further inspection, 32 articles were included in the final meta-analysis, while 27 studies were excluded on the following grounds: 3 were focused on pediatric populations; 4 were unavailable in full text; 2 were unavailable in English; 11 were conducted on populations outside our regions of interest; 6 did not have a non-IBD control group; and 1 study was unclear in the results pertaining to IBD subtypes. A detailed flow diagram of our study selection process is shown in Fig. 1. The main characteristics of the included studies are listed in Table 1 . They include all 3 regions of interest: East Asia, Europe and the Mediterranean [22,35-65]. In our meta-analysis, 22 studies were case-control studies, while 10 were cohort studies. Of the 32 studies, 17 of them $(53.12 \%)$ scored $\geq 7$ on the Newcastle-Ottawa scale. In total, the 32 studies in this meta-analysis included 4607 IBD cases and 4666 controls.

\section{Effect size estimates (RR) and contrasts (RRR)}

In this meta-analysis, $24.33 \%$ of patients with IBD had H. pylori infection, compared to $43.12 \%$ in the non-IBD control group. A comparison of the full and reduced metaregression models showed no evidence of a significant subtype $\times$ region interaction (likelihood ratio test: $\lambda_{\mathrm{LR}}=1.07$; $\mathrm{P}=0.59$ ) on $\mathrm{RR}$. We therefore used the reduced version as the main model in this study. Sensitivity analysis showed that the predictions (RR) and contrasts (RRR) from this model were generally robust in response to the omission of any particular study (see supplementary material). This model exhibited moderate heterogeneity $\left(I^{2}=48.74 \%\right.$; Cochran's Q test: $\left.\chi_{49}^{2}=100.55, \mathrm{P}<0.001\right)$. Both region (Wald test: $\chi_{2}^{2}=7.73$, $\mathrm{P}=0.02$ ) and subtype (Wald test: $\chi_{1}^{2}=13.98, \mathrm{P}<0.001$ ) were both statistically significant as main effects (omnibus Wald test of both moderators $\chi_{3}^{2}=21.87, \mathrm{P}<0.001$ ).

Pooled RR's were $<1$ for all subtype and region combinations, implying a negative association between $H$. pylori and IBD. Model predictions (i.e., summary effects presented as RR with corresponding lower and upper 95\% confidence bounds) were as follows: CD-East Asia 0.43 (0.36-0.52); CD-Europe 0.51 (0.42-0.61); CD-Mediterranean 0.62 (0.50-0.76); UC-East Asia 0.55 (0.46-0.66); UC-Europe 0.65 (0.54-0.78); and UCMediterranean 0.79 (0.64-0.96) (Fig. 2). The protective effect of H. pylori appears to be greatest in East Asian regions (lowest RR), followed by European regions, while Mediterranean regions have the least protective effect in both CD and UC subtypes. In addition, the protective effect seems to be greater for CD than for UC across all regions. Although the pooled RRs were $<1$ for all subtype and region combinations, the RR was $28 \%$ (9\%, $50 \%)$ greater for UC vs. $\mathrm{CD}(\mathrm{RRR}=1.28[1.09,1.50], \mathrm{P}<0.001)$ and $43 \%[4 \%, 96 \%]$ greater for Mediterranean vs. East Asia $(\mathrm{RRR}=1.43$ [1.04, 1.96], $\mathrm{P}=0.02)$ (Fig. 3). Pooled RRs were 18\% $(-13 \%, 60 \%)$ greater for Europe vs. East Asia $(R R R=1.18[0.87$; $1.60], \mathrm{P}=0.48)$ and $21 \%$ [-13\%, 68\%] greater for Mediterranean vs. Europe $[\mathrm{RRR}=1.21[0.87 ; 1.68], \mathrm{P}=0.42)$, though these differences were not statistically significant.(Fig. 3).

\section{Publication bias}

A funnel plot showed slight asymmetry (Fig. 4), suggestive of possible small-study bias, also suggested by the pseudo Egger regression test: $(\mathrm{Z}=-6.35 ; \mathrm{P}<0.001)$. However, the contour-enhanced funnel plot shows approximate symmetry and suggests that publication bias is unlikely (Fig. 5).

\section{Discussion}

This is the fifth meta-analysis studying the relationship between $H$. pylori and IBD [13-16]. Our meta-analysis has 


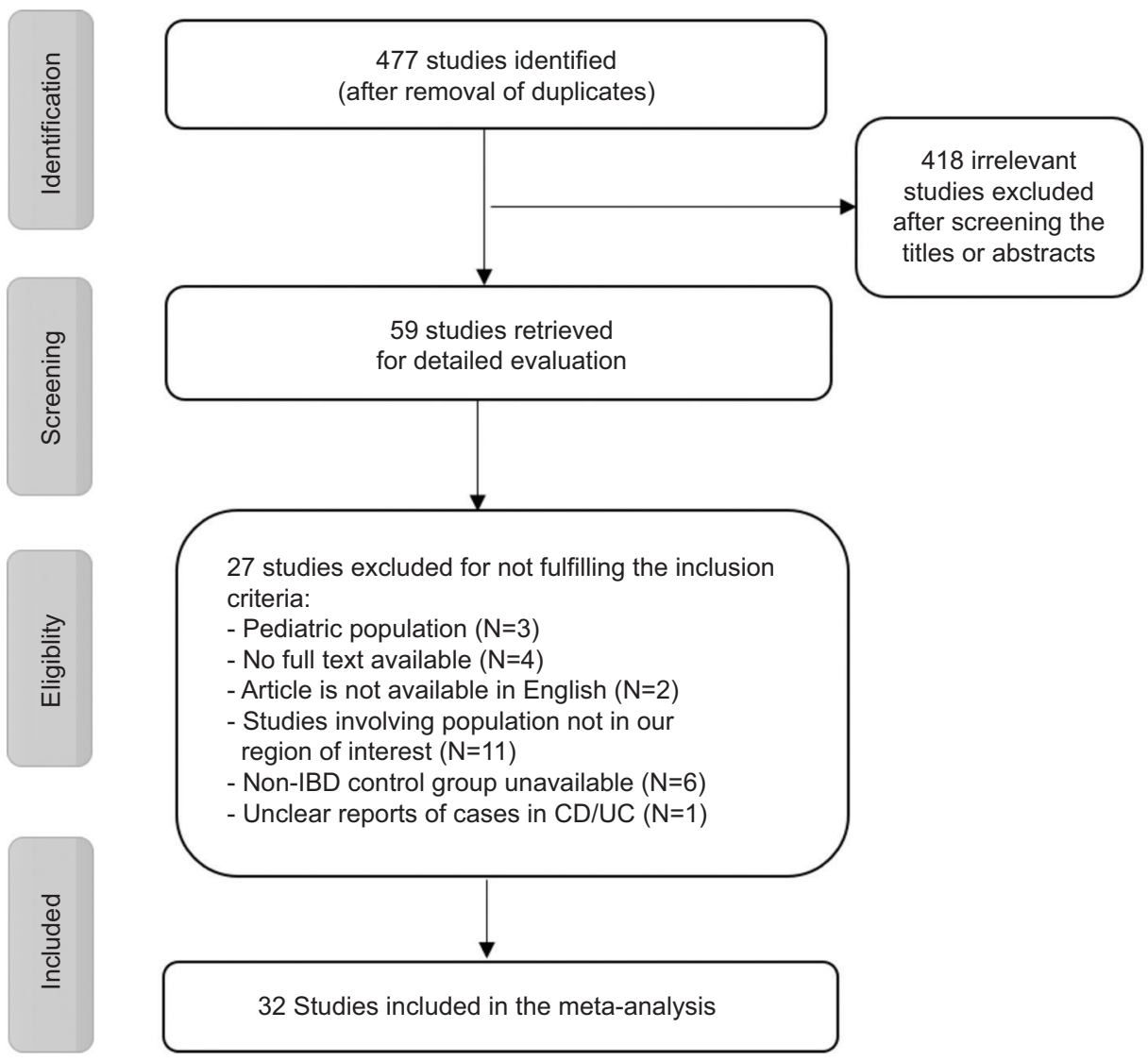

Figure 1 Study flow diagram showing identification, screening and eligibility stages which resulted in 32 studies being included in our meta-analysis $N$; number

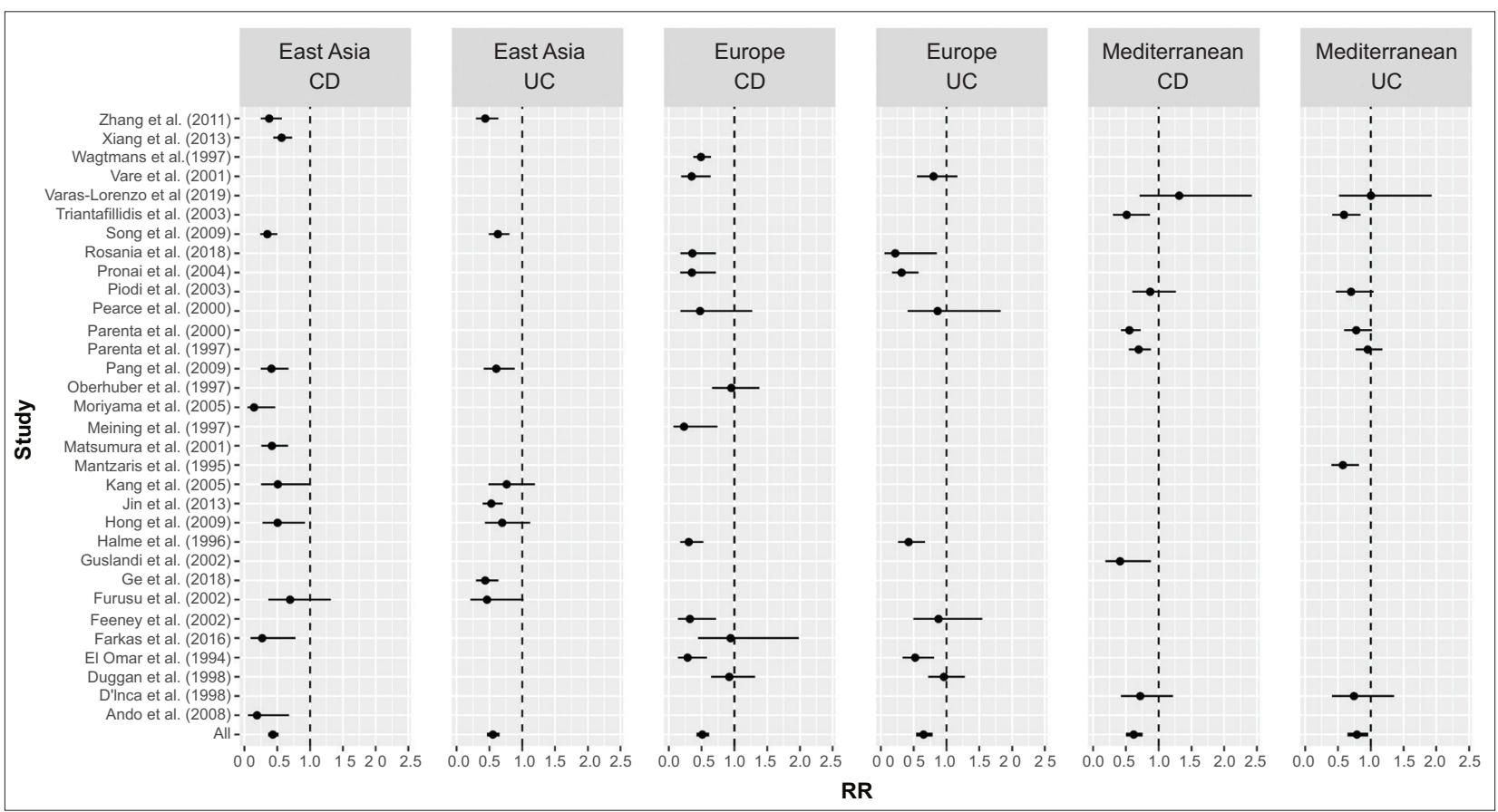

Figure 2 Relative risks (RR) and 95\% confidence intervals for each primary study by region and subtype $(\mathrm{CD}=\mathrm{Crohn}$ 's disease; UC = ulcerative colitis. The pooled summary effects are shown at the base of the plot aligned with 'All'. Vertical dashed lines are shown at $\mathrm{RR}=1$ to indicate the null effect (confidence intervals that do not include 1 are statistically significant at $\mathrm{P}<0.05$ ) 







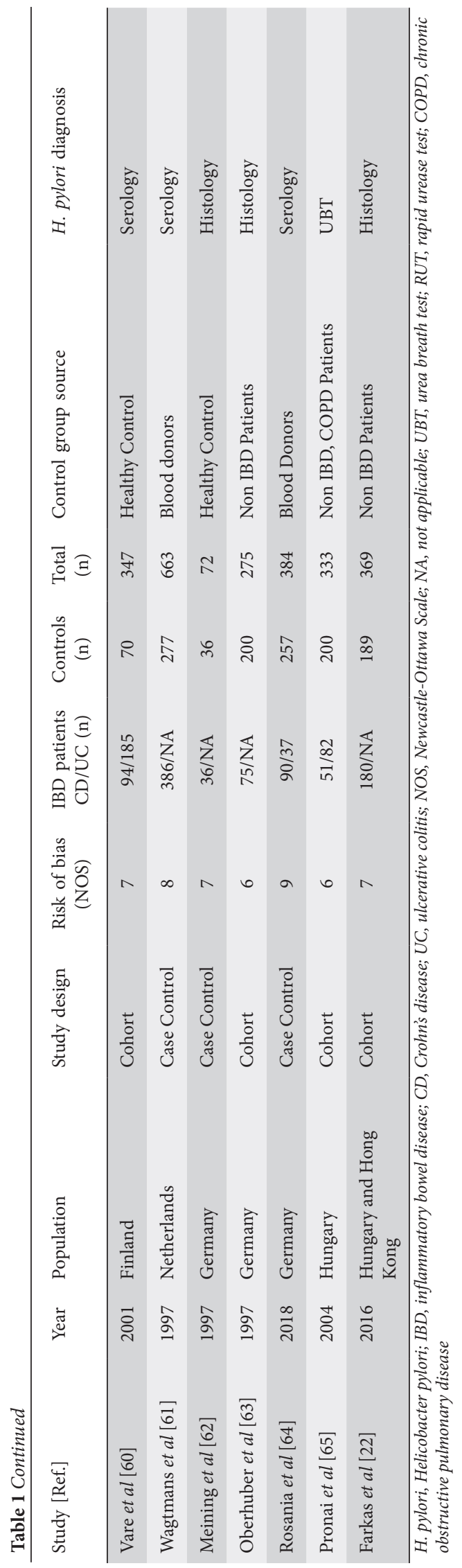

extended previous work by simultaneously quantifying the impact of geographic region (East Asia, non-Mediterranean Europe, Mediterranean) and IBD subtype (CD, UC) on the association between $H$. pylori and IBD. We found significant effects of both region and IBD subtype, though there was no evidence to suggest these effects were interactive, i.e., that the difference between IBD subtypes varied across regions, or vice versa. Although the RR for all region and subtype combinations suggest that $H$. pylori infection has a protective effect against the development of IBD, the RR was $28 \%$ greater for UC than CD (pooled across all regions), and 43\% greater for Mediterranean compared to East Asian regions (pooled across subtypes).

The protective effect of $H$. pylori infection on IBD incidence has strong support [40-43,48,53,58,60,65], despite some studies suggesting that a lower $H$. pylori infection rate may be an artefact of IBD treatment (e.g., sulfasalazine, mesalazine, corticosteroids, antibiotics, etc.) eradicating $H$. pylori in some patients $[47,51,52]$. IBD is known to initiate an increase in type $1 \mathrm{~T}$ helper lymphocyte (Th1) and/or T helper 17 cells (Th17), resulting in higher inflammatory factors [66]. Moreover, CD patients have a tendency for selective activation of Th1- and Th17-related cytokines, which possibly explains the greater protective effect of $H$. pylori in CD compared to UC [16]. This trend was suggested by previous meta-analyses [13-16], although we are the first to confirm the effect using formal hypothesis tests.

Previous meta-analyses have suggested that the beneficial effects of $H$. pylori on the risk of IBD are greater for eastern than for western populations [16]. A possible explanation is the greater relative abundance of the seropositive CagA H. pylori strain in East Asian compared to western populations [23]. It has been suggested that expression of CagA might increase the production of beta-defensins, thought to play a protective role in IBD pathogenesis [23]. Another possible theory is that during $H$. pylori infection, the response of the type $2 \mathrm{~T}$ helper cell 2 (Th2) cytokine is dependent on the presence of CagA strain [23]. Indeed, this specific response has been shown to be protective against gut inflammation [23].

Genetic disparities in the patients themselves might also explain the variation in the protective effect of $H$. pylori across geographic regions. A possible hypothesis is that IBD in East Asian populations is less likely to be attributed to genetic factors compared to those in the west. In Asian populations, a family history of IBD is not frequently observed, relative to counterparts in Europe or North America [24,67]. One of the most studied genes in IBD heritability in the West is the nucleotide-binding oligomerization domain-containing protein 2 (NOD2) [67,68]. Genetic studies have found no evidence of a correlation between NOD2 and IBD in Asian populations [69-71]. However, new NOD2 mutations such as (JW1) have been reported in Malaysian populations, while increased expression of (P268S) was reported in Han Chinese and Indian populations [69-71]. In a genome-wide association study (GWAS) that investigated the role of single nucleotide polymorphism (SNP), the autophagy-related 16-like 1 gene (ATG16L1) and immunity-related GTPase family $M$ gene (IRGM) were found to potentially increase susceptibility 


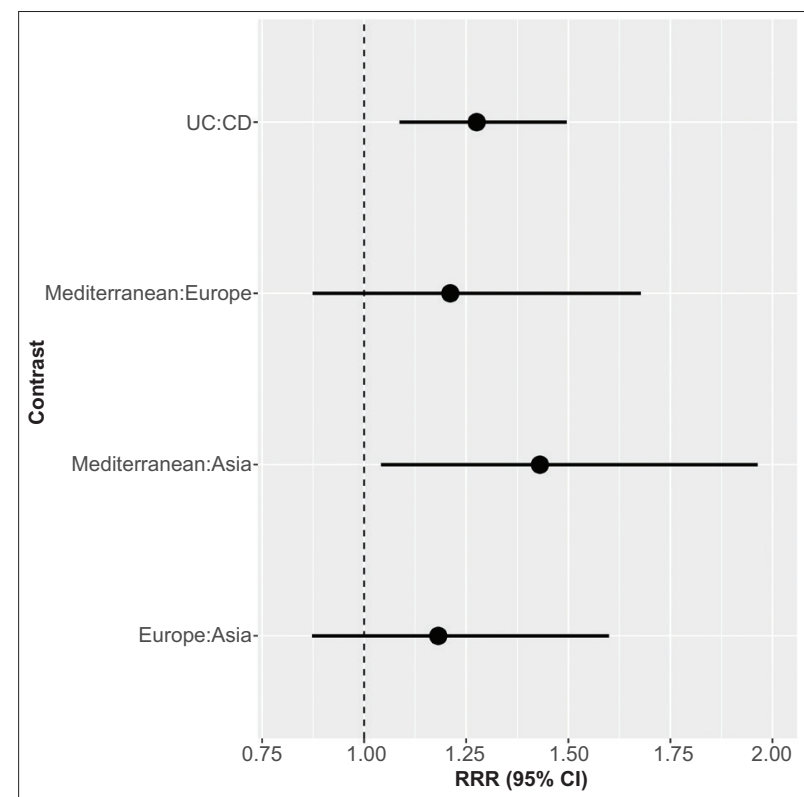

Figure 3 Ratios of relative risks (RRR) and 95\% confidence intervals for pre-specified contrasts of moderator variables (region and subtype) included in our meta-regression model. RRR represent average effects, pooled over levels of the other moderator in the model. Confidence intervals are adjusted for simultaneous inference using the single-step method. The vertical dashed line at $R R R=1$ indicates the null effect (confidence intervals that do not include 1 are statistically significant at $\mathrm{P}<0.05$ )

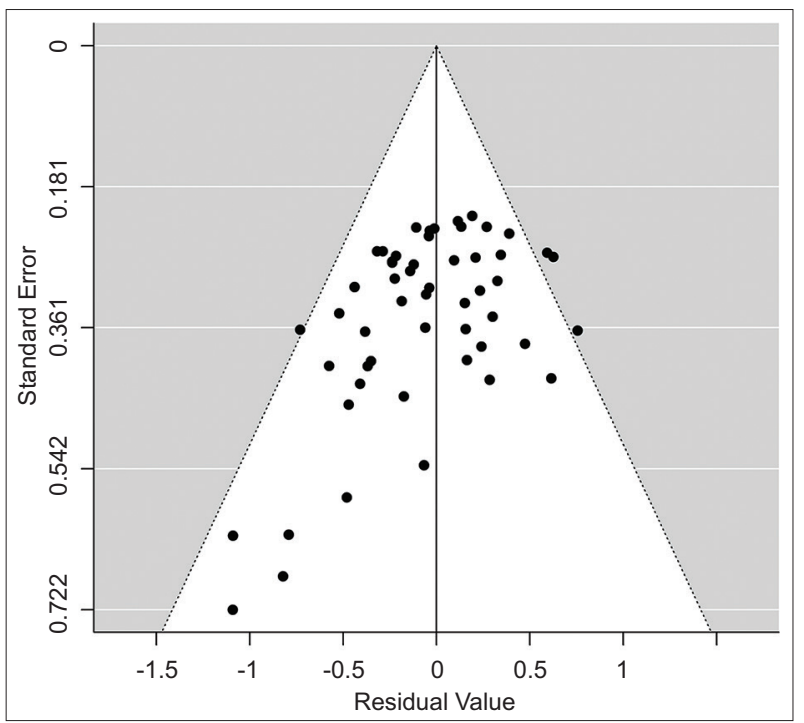

Figure 4 Standard funnel plot for all studies included in our metaregression model. The cluster of studies on the lower left side might be suggestive of small study bias

for IBD in Western populations [72]. Similar studies on Asian populations have shown mixed results in Korea and Japan [67,72]. Currently, more than 230 SNPs have been linked to IBD through GWAS, though their exact roles and mechanism of action in IBD pathogenesis are yet to be fully described $[67,73]$.

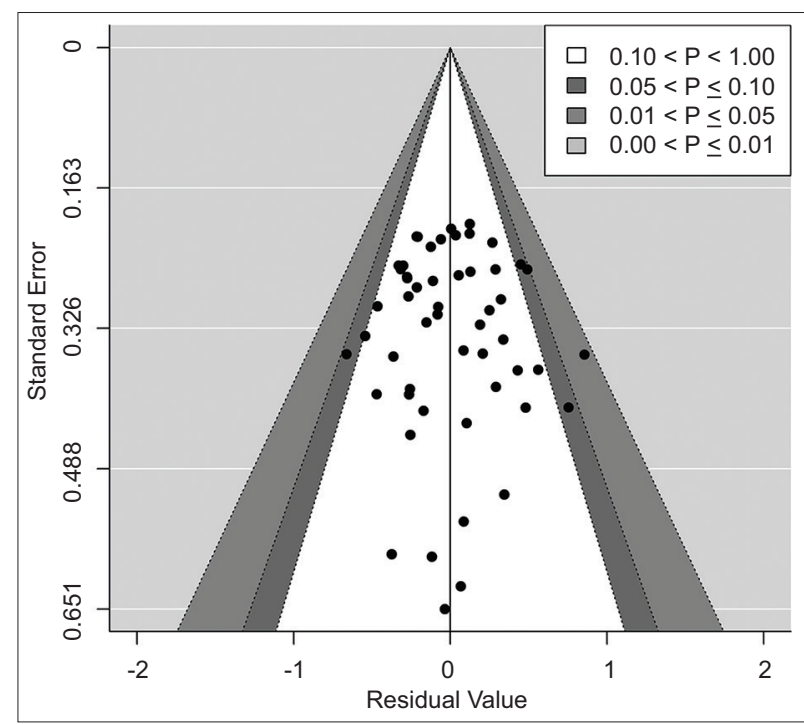

Figure 5 Contour-enhanced funnel plot for all studies included in our meta-regression model. The plot is approximately symmetrical and is not indicative of publication bias. All of the smaller studies (larger standard error towards base of plot) lie within $0.10<\mathrm{P}<1.00$

Regional variation in the protective effect of $H$. pylori on IBD might also be ascribed to environmental and socioeconomic factors, such as the role of diet. The western diet is thought to be particularly conducive to IBD, owing to the low intake of fiber and high intake of refined carbohydrate and processed meat [18,74]. Indeed, as IBD incidence continues to rise in Asia [7], many studies have suggested that this could be the result of Asian populations adopting the western diet [18,74-76]. The Mediterranean diet, plant-based diet and semi-vegetarian diet have been shown by some studies to help alleviate symptoms of IBD and keep IBD patients in remission $[17,77,78]$. It is noteworthy that the Mediterranean diet has also been reported to reduce inflammation and improve microbiota in IBD patients [17-19]. Interestingly, our metaanalysis found that the protective effect of $H$. pylori against IBD in Mediterranean populations is less than in both East Asian and European counterparts (though only the former difference was statistically significant). Although this might suggest that the protective effect of diet is questionable, our meta-analysis did not explicitly incorporate dietary information and any putative effects of diet might be confounded in such a broadscale analysis. Nevertheless, this does raise the need for further research into the role of diet on incidence of IBD. Although numerous studies have been conducted on recommended diets for prevention of IBD, there is still no consensus as to which is optimal [79]. Robust clinical trials have been limited by challenges such as defining the diet intervention, blinding, measuring intake and adherence over an extended period of time [79].

In conclusion, our meta-analysis supports previous findings of a protective effect of $H$. pylori infection on the risk of IBD, though we are the first to confirm that this protective effect is significantly stronger for CD compared to UC and for East Asian compared to Mediterranean populations. Our 
study is not without limitations and further primary research is warranted to evaluate genetic variability, pathogenesis, immunologic response, and environmental and dietary factors. A potential avenue for extending our study involves broadening the inclusion criteria to gain further insight into the regional variation of the protective effects of $H$. pylori on IBD.

\section{Summary Box}

\section{What is already known:}

- Helicobacter pylori (H. pylori) infection appears to have a protective effect against inflammatory bowel disease (IBD)

- H. pylori appears to be more protective against Crohn's disease (CD) than ulcerative colitis (UC), and in East Asian vs. western regions, though these have yet to be formally compared using hypothesis tests

\section{What the new findings are:}

- Our meta-analysis found a significant negative association between $H$. pylori infection and IBD, which varies by both IBD subtype (CD, UC) and geographic region (East Asia, Europe, Mediterranean)

- H. pylori infection provides significantly more protection against CD compared with UC

- H. pylori infection provides significantly more protection against IBD in East Asian compared to Mediterranean regions

\section{Acknowledgements}

We would like to thank Dr Wolfgang Viechtbauer for his kind advice on use of the R package "metafor", Levas Mejus and Norasikin Repan from Newcastle University Medicine Malaysia Learning Resources Centre for their support in reference management software

\section{References}

1. Singh P, Ananthakrishnan A, Ahuja V. Pivot to Asia: inflammatory bowel disease burden. Intest Res 2017;15:138-141.

2. Burisch J, Munkholm P. The epidemiology of inflammatory bowel disease. Scand J Gastroenterol 2015;50:942-951.

3. Ng SC, Shi HY, Hamidi N, et al. Worldwide incidence and prevalence of inflammatory bowel disease in the 21st century: a systematic review of population-based studies. Lancet 2018;390:2769-2778.

4. Kaplan GG. The global burden of IBD: from 2015 to 2025. Nat Rev Gastroenterol Hepatol 2015;12:720-727.
5. GBD 2017 Inflammatory Bowel Disease Collaborators. The global, regional, and national burden of inflammatory bowel disease in 195 countries and territories, 1990-2017: a systematic analysis for the Global Burden of Disease Study 2017. Lancet Gastroenterol Hepatol 2020;5:17-30.

6. Ng WK, Wong SH, Ng SC. Changing epidemiological trends of inflammatory bowel disease in Asia. Intest Res 2016;14:111-119.

7. Thia KT, Loftus EV Jr, Sandborn WJ, Yang SK. An update on the epidemiology of inflammatory bowel disease in Asia. Am J Gastroenterol 2008;103:3167-3182.

8. Knowles SR, Graff LA, Wilding H, Hewitt C, Keefer L, MikockaWalus A. Quality of life in inflammatory bowel disease: a systematic review and meta-analyses-Part I. Inflamm Bowel Dis 2018;24:742-751.

9. Zhang C, Byrne G, Lee T, Singer J, Giustini D, Bressler B . Incidence of suicide in inflammatory bowel disease: a systematic review and meta-analysis. J Can Assoc Gastroenterol 2018;1:107-114.

10. Ryan E, McNicholas D, Creavin B, Kelly ME, Walsh T, Beddy D. Sarcopenia and inflammatory bowel disease: a systematic review. Inflamm Bowel Dis 2019;25:67-73.

11. Axelrad JE, Lichtiger S, Yajnik V. Inflammatory bowel disease and cancer: The role of inflammation, immunosuppression, and cancer treatment. World J Gastroenterol 2016;22:4794-4801.

12. Zamani M, Ebrahimtabar F, Zamani V, et al. Systematic review with meta-analysis: the worldwide prevalence of Helicobacter pylori infection. Aliment Pharmacol Ther 2018;47:868-876.

13. Luther J, Dave M, Higgins PD, Kao JY. Association between Helicobacter pylori infection and inflammatory bowel disease: a meta-analysis and systematic review of the literature. Inflamm Bowel Dis 2010;16:1077-1084

14. Rokkas T, Rokka A, Portincasa P. A systematic review and metaanalysis of the role of Helicobacter pylori eradication in preventing gastric cancer. Ann Gastroenterol 2017;30:414-423.

15. Wu XW, Ji HZ, Yang MF, Wu L, Wang FY. Helicobacter pylori infection and inflammatory bowel disease in Asians: a metaanalysis. World J Gastroenterol 2015;21:4750-4756.

16. Castaño-Rodríguez N, Kaakoush NO, Lee WS, Mitchell HM. Dual role of Helicobacter and Campylobacter species in IBD: a systematic review and meta-analysis. Gut 2017;66:235-249.

17. Marlow G, Ellett S, Ferguson IR, et al. Transcriptomics to study the effect of a Mediterranean-inspired diet on inflammation in Crohn's disease patients. Hum Genomics 2013;7:24.

18. Haskey N, Gibson DL. An examination of diet for the maintenance of remission in inflammatory bowel disease. Nutrients 2017;9.

19. Reddavide R, Rotolo O, Caruso MG, et al. The role of diet in the prevention and treatment of inflammatory bowel diseases. Acta Biomed 2018;89:60-75.

20. Kim DH, Cheon JH. Pathogenesis of inflammatory bowel disease and recent advances in biologic therapies. Immune Netw 2017; 17:25-40.

21. Ng SC. Emerging trends of inflammatory bowel disease in Asia. Gastroenterol Hepatol (N Y) 2016;12:193-196.

22. Farkas K, Chan H, Rutka M, et al. Gastroduodenal involvement in asymptomatic Crohn's disease patients in two areas of emerging disease: Asia and Eastern Europe. J Crohns Colitis 2016;10:1401-1406.

23. Tepler A, Narula N, Peek RM Jr, et al. Systematic review with meta-analysis: association between Helicobacter pylori CagA seropositivity and odds of inflammatory bowel disease. Aliment Pharmacol Ther 2019;50:121-131.

24. Yang SK. How does the epidemiology of inflammatory bowel disease differ between east and west? A Korean perspective. Inflamm Intest Dis 2017;2:95-101.

25. Moher D, Liberati A, Tetzlaff J, Altman DG; PRISMA Group. Preferred reporting items for systematic reviews and metaanalyses: the PRISMA statement. BMJ 2009;339:b2535.

26. Wells G, Shea B, O'Connell D, et al. The Newcastle-Ottawa Scale 
(NOS) for assessing the quality of nonrandomised studies in meta-analyses. In. 2013. Wells G, Shea B, O'Connell D et al. The Newcastle-Ottawa Scale (NOS) for assessing the quality of nonrandomised studies in meta-analyses. In. Ottawa, Canada. : University of Ottawa 2013; Available from: http://www.ohri.ca/ programs/clinical_epidemiology/oxford.asp [Accessed 18 May 2020].

27. Lo CK, Mertz D, Loeb M. Newcastle-Ottawa Scale: comparing reviewers' to authors' assessments. BMC Med Res Methodol 2014;14:45.

28. $\mathrm{R}$ Core Team. R: A language and environment for statistical computing. In $\mathrm{R}$ version 3.6.0 Edition. Vienna, Austria. : $R$ Foundation for Statistical Computing 2013; Available from: https:// www.r-project.org/ [Accessed 18 May 2020].

29. Viechtbauer W. Conducting meta-analyses in $\mathrm{R}$ with the metafor package. J Stat Softw 2010;36:1-48.

30. Wickham H. ggplot2: Elegant Graphics for Data Analysis. New York: Springer-Verlag; 2016.

31. Konstantopoulos S. Fixed effects and variance components estimation in three-level meta-analysis. Res Synth Methods 2011;2:61-76.

32. Osborne JW. Best practice in quantitative methods. California: SAGE Inc.; 2008.

33. Cochran W. The combination of estimates from different experiments. Biometrics 1954;10:101-129.

34. Higgins JP, Thompson SG, Deeks JJ, Altman DG. Measuring inconsistency in meta-analyses. BMJ 2003;327:557-560.

35. Matsumura M, Matsui T, Hatakeyama S, et al. Prevalence of Helicobacter pylori infection and correlation between severity of upper gastrointestinal lesions and H. pylori infection in Japanese patients with Crohn's disease. J Gastroenterol 2001;36:740-747.

36. Furusu H, Murase K, Nishida Y, et al. Accumulation of mast cells and macrophages in focal active gastritis of patients with Crohn's disease. Hepatogastroenterology 2002;49:639-643.

37. Moriyama T, Matsumoto T, Jo Y, et al. Mucosal proinflammatory cytokine and chemokine expression of gastroduodenal lesions in Crohn's disease. Aliment Pharmacol Ther 2005;21 (Suppl 2):85-91.

38. Ando T, Watanabe $\mathrm{O}$, Ishiguro $\mathrm{K}$, et al. Relationships between Helicobacter pylori infection status, endoscopic, histopathological findings, and cytokine production in the duodenum of Crohn's disease patients. J Gastroenterol Hepatol 2008;23(Suppl 2):S193-S197.

39. Kang MS, Park DI, Park JH, et al. [Bamboo joint-like appearance of stomach in Korean patients with Crohn's disease]. Korean J Gastroenterol 2006;48:395-400.

40. Song MJ, Park DI, Hwang SJ, et al. [The prevalence of Helicobacter pylori infection in Korean patients with inflammatory bowel disease, a multicenter study]. Korean $J$ Gastroenterol 2009;53:341-347.

41. Hong CH, Park DI, Choi WH, et al. [The clinical usefulness of focally enhanced gastritis in Korean patients with Crohn's disease]. Korean J Gastroenterol 2009;53:23-28.

42. Pang Z, Li MF, Huangfu Z, et al. Analysis of Helicobacter pylori infection in Chinese Han inflammatory bowel disease. Shi Jie Hua Ren Xiao Hua Za Zhi 2009;17:3661-3665.

43. Zhang S, Zhong B, Chao K, et al. Role of Helicobacter species in Chinese patients with inflammatory bowel disease. J Clin Microbiol 2011;49:1987-1989.

44. Jin X, Chen YP, Chen SH, Xiang Z. Association between Helicobacter pylori infection and ulcerative colitis-a case control study from China. Int J Med Sci 2013;10:1479-1484.

45. Xiang Z, Chen YP, Ye YF, et al. Helicobacter pylori and Crohn's disease: a retrospective single-center study from China. World $J$ Gastroenterol 2013;19:4576-4581.

46. Ge YF, Guan X, Jiang XJ. Clinical significance of Helicobacter pylori in the development of ulcerative colitis. Shi Jie Hua Ren Xiao Hua Za Zhi 2018;26:1071-1076.

47. Parente F, Molteni P, Bollani S, et al. Prevalence of Helicobacter pylori infection and related upper gastrointestinal lesions in patients with inflammatory bowel diseases. A cross-sectional study with matching. Scand J Gastroenterol 1997;32:1140-1146.

48. D’Incà R, Sturniolo G, Cassaro M, et al. Prevalence of upper gastrointestinal lesions and Helicobacter pylori infection in Crohn's disease. Dig Dis Sci 1998;43:988-992.

49. Parente F, Cucino C, Bollani S, et al. Focal gastric inflammatory infiltrates in inflammatory bowel diseases: prevalence, immunohistochemical characteristics, and diagnostic role. Am J Gastroenterol 2000;95:705-711.

50. Guslandi M, Fanti L, Testoni PA. Helicobacter pylori seroprevalence in Crohn's disease: lack of influence by pharmacological treatment. Hepatogastroenterology 2002;49:1296-1297.

51. Piodi LP, Bardella M, Rocchia C, Cesana BM, Baldassarri A, Quatrini M. Possible protective effect of 5-aminosalicylic acid on Helicobacter pylori infection in patients with inflammatory bowel disease. J Clin Gastroenterol 2003;36:22-25.

52. Mantzaris GJ, Archavlis E, Zografos C, Zavos K, Petraki K, Triadaphyllou G. Low prevalence of Helicobacter pylori in inflammatory bowel disease: association with sulfasalazine. Am J Gastroenterol 1995;90:1900.

53. Triantafillidis JK, Gikas A, Apostolidiss N, Merikas E, Mallass E, Peros G. The low prevalence of Helicobacter infection in patients with inflammatory bowel disease could be attributed to previous antibiotic treatment. Am J Gastroenterol 2003;98:1213-1214.

54. Varas Lorenzo M, Muñoz Agel F, Sanchez-Vizcaino Mengual E. Eradication of Helicobacter pylori in patients with inflammatory bowel disease for prevention of recurrences-impact on the natural history of the disease. Eurasian J Med Oncol 2019;3:59-65.

55. el-Omar E, Penman I, Cruikshank G, et al. Low prevalence of Helicobacter pylori in inflammatory bowel disease: association with sulphasalazine. Gut 1994;35:1385-1388.

56. Duggan AE, Usmani I, Neal KR, Logan RF. Appendicectomy, childhood hygiene, Helicobacter pylori status, and risk of inflammatory bowel disease: a case control study. Gut 1998;43:494-498.

57. Pearce CB, Duncan HD, Timmis L, Green JR. Assessment of the prevalence of infection with Helicobacter pylori in patients with inflammatory bowel disease. Eur J Gastroenterol Hepatol 2000;12:439-443.

58. Feeney MA, Murphy F, Clegg AJ, Trebble TM, Sharer NM, Snook JA. A case-control study of childhood environmental risk factors for the development of inflammatory bowel disease. Eur $J$ Gastroenterol Hepatol 2002;14:529-534.

59. Halme L, Rautelin H, Leidenius M, Kosunen TU. Inverse correlation between Helicobacter pylori infection and inflammatory bowel disease. J Clin Pathol 1996;49:65-67.

60. Väre PO, Heikius B, Silvennoinen JA, et al. Seroprevalence of Helicobacter pylori infection in inflammatory bowel disease: is Helicobacter pylori infection a protective factor? Scand J Gastroenterol 2001;36:1295-1300.

61. Wagtmans MJ, Witte AM, Taylor DR, et al. Low seroprevalence of Helicobacter pylori antibodies in historical sera of patients with Crohn's disease. Scand J Gastroenterol 1997;32:712-718.

62. Meining A, Bayerdörffer E, Bastlein E, et al. Focal inflammatory infiltrations in gastric biopsy specimens are suggestive of Crohn's disease. Crohn's Disease Study Group, Germany. Scand J Gastroenterol 1997;32:813-818.

63. Oberhuber G, Püspök A, Oesterreicher C, et al. Focally enhanced gastritis: a frequent type of gastritis in patients with Crohn's disease. Gastroenterology 1997;112:698-706.

64. Rosania R, Von Arnim U, Link A, et al. Helicobacter pylori eradication therapy is not associated with the onset of inflammatory 
bowel diseases. A case-control study. J Gastrointestin Liver Dis 2018;27:119-125.

65. Prónai L, Schandl L, Orosz Z, Magyar P, Tulassay Z. Lower prevalence of Helicobacter pylori infection in patients with inflammatory bowel disease but not with chronic obstructive pulmonary disease - antibiotic use in the history does not play a significant role. Helicobacter 2004;9:278-283.

66. Yu Y, Zhu S, Li P, Min L, Zhang S. Helicobacter pylori infection and inflammatory bowel disease: a crosstalk between upper and lower digestive tract. Cell Death Dis 2018;9:961.

67. Park SC, Jeen YT. Genetic studies of inflammatory bowel diseasefocusing on Asian patients. Cells 2019;8.

68. Ek WE, D'Amato M, Halfvarson J. The history of genetics in inflammatory bowel disease. Ann Gastroenterol 2014;27:294-303.

69. Turpin W, Goethel A, Bedrani L, Croitoru Mdcm K. Determinants of IBD heritability: genes, bugs, and more. Inflamm Bowel Dis 2018;24:1133-1148.

70. Kedia S, Ahuja V. Epidemiology of inflammatory bowel disease in India: The Great Shift East. Inflamm Intest Dis 2017;2:102-115.

71. Ng SC, Tsoi KK, Kamm MA, et al. Genetics of inflammatory bowel disease in Asia: systematic review and meta-analysis. Inflamm Bowel Dis 2012;18:1164-1176.
72. Verstockt B, Smith KG, Lee JC. Genome-wide association studies in Crohn's disease: past, present and future. Clin Transl Immunology 2018;7:e1001.

73. de Lange KM, Moutsianas L, Lee JC, et al. Genome-wide association study implicates immune activation of multiple integrin genes in inflammatory bowel disease. Nat Genet 2017;49:256-261.

74. Konkel L. Inflammatory bowel disease in Asia: a second chance at uncovering environmental factors. Environ Health Perspect 2016;124:A49-A54.

75. Knight-Sepulveda K, Kais S, Santaolalla R, Abreu MT. Diet and inflammatory bowel disease. Gastroenterol Hepatol (N Y) 2015;11:511-520.

76. Limdi JK. Dietary practices and inflammatory bowel disease. Indian J Gastroenterol 2018;37:284-292.

77. Kakodkar S, Mutlu EA. Diet as a therapeutic option for adult inflammatory bowel disease. Gastroenterol Clin North Am 2017;46:745-767.

78. Chiba M, Ishii H, Komatsu M. Recommendation of plant-based diets for inflammatory bowel disease. Transl Pediatr 2019;8:23-27.

79. Lewis JD, Albenberg L, Lee D, Kratz M, Gottlieb K, Reinisch W. The importance and challenges of dietary intervention trials for inflammatory bowel disease. Inflamm Bowel Dis 2017;23:181-191. 


\section{Supplementary material}

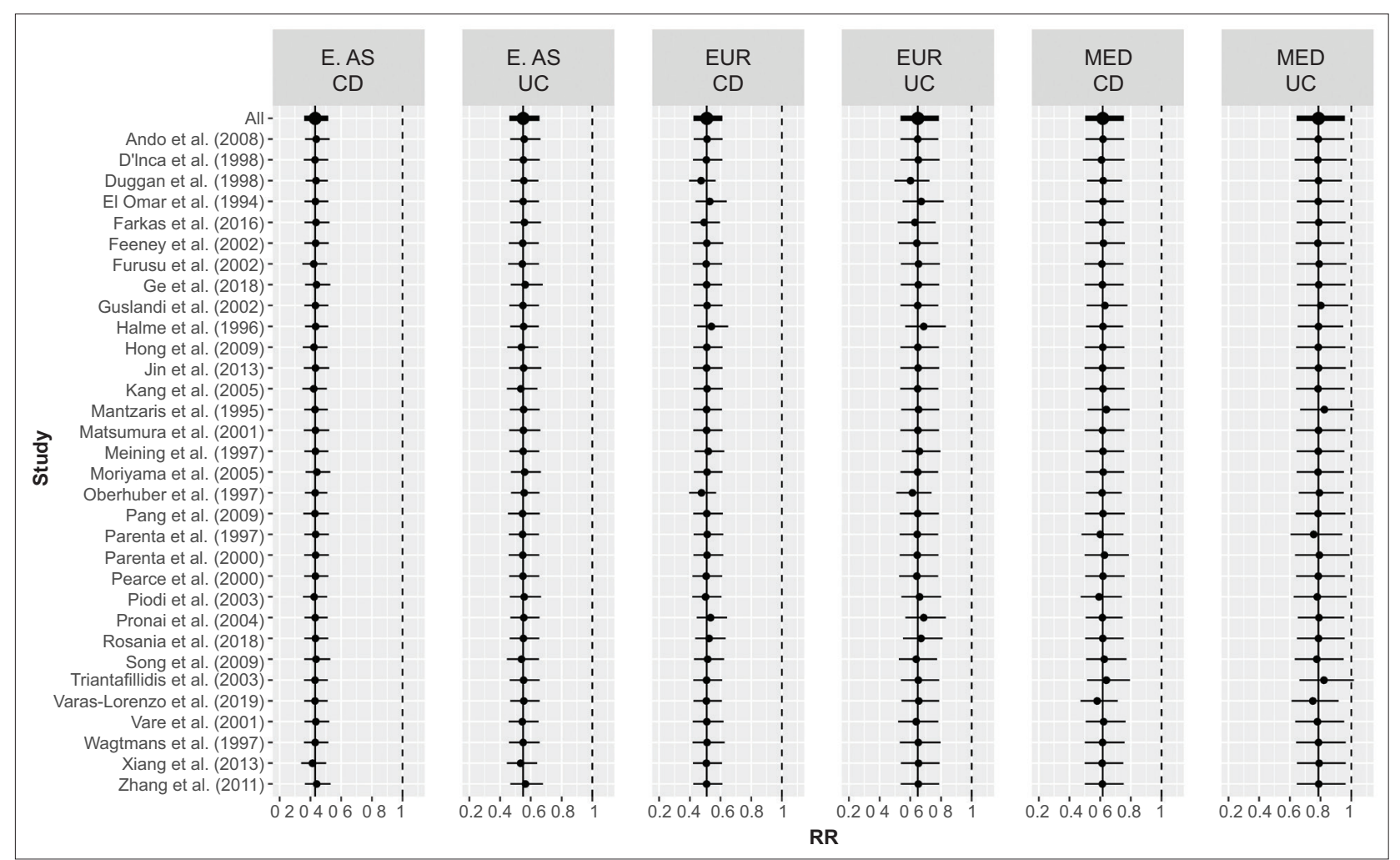

Supplementary Figure 1 Leave-one-out sensitivity analysis for model predictions (summary effects expressed as relative risk [RR] with 95\% confidence intervals) by region and subtype. The uppermost RR corresponds to summary effects presented in the paper, including all 32 studies in the meta-regression model. The remaining RR are model predictions with the corresponding study on the y-axis omitted. Vertical dashed lines are shown at $\mathrm{RR}=1$ to indicate the null effect (confidence intervals that do not include 1 are statistically significant at $\mathrm{P}<0.05$ ). The solid vertical lines represent the summary effect for all studies to help illustrate the displacement when each study is omitted. Under no circumstances does omitting a study result in a significant deviation from the overall summary effect. In 2 instances, the summary effect for Mediterranean-UC becomes (just) non-significant (Mantzaris et al 1995 and Triantafillidis et al 2003) 


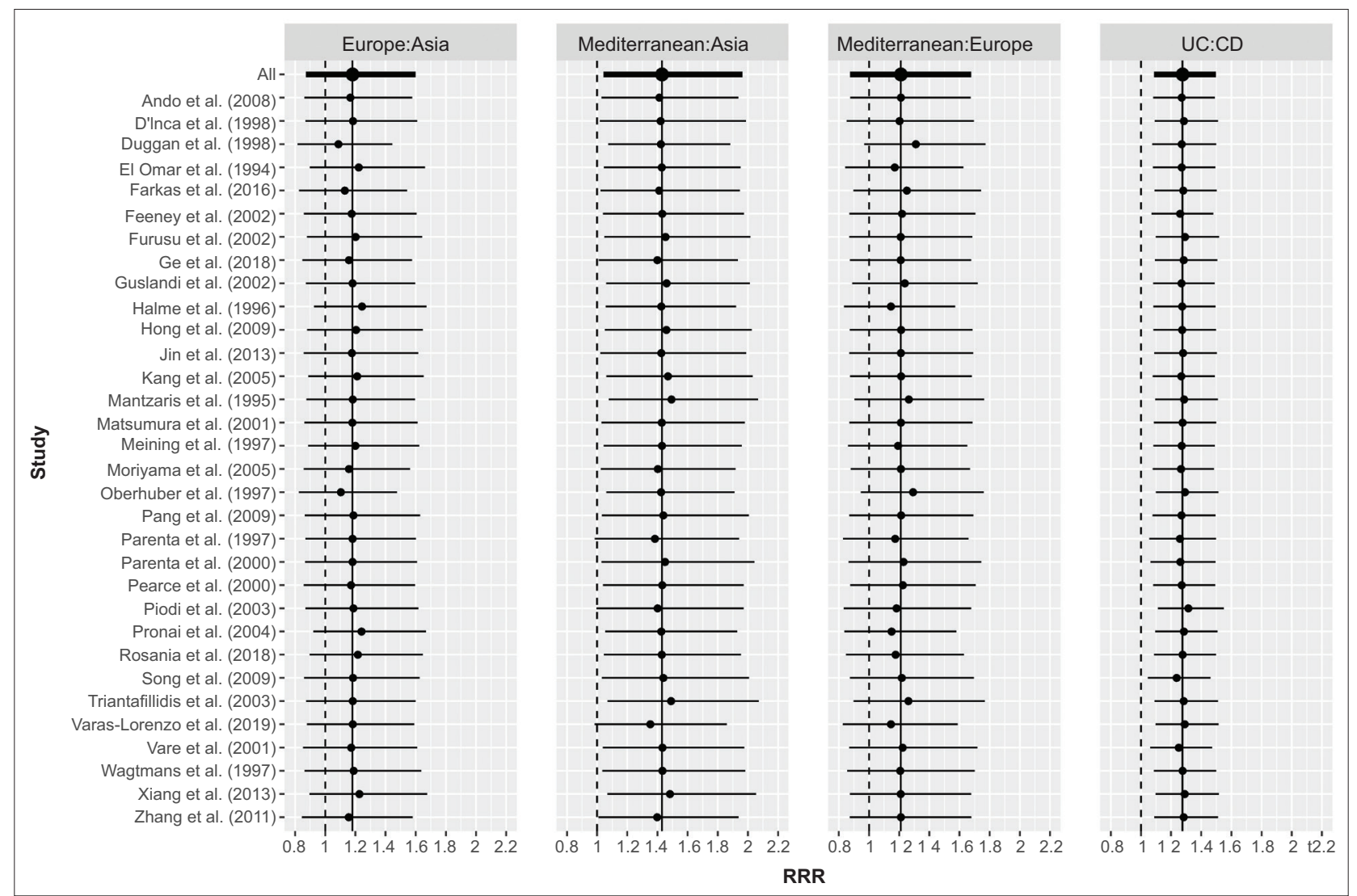

Supplementary Figure 2 Leave-one-out sensitivity analysis for model contrasts (expressed as ratios of relative risk [RRR] with 95\% confidence intervals) for pre-specified contrasts of moderator variables (region and subtype) included in our meta-regression model. Confidence intervals are adjusted for simultaneous inference using the single-step procedure. RRR represent average effects, pooled over levels of the other moderator in the model. The uppermost RRR corresponds to those presented in the paper, including all 32 studies in the meta-regression model. The remaining RRR are contrasts with the corresponding study on the $y$-axis omitted. Vertical dashed lines are shown at $R R R=1$ to indicate the null effect (confidence intervals that do not include 1 are statistically significant at $\mathrm{P}<0.05)$. The solid vertical lines represent the RRR for all studies to help illustrate the displacement when each study is omitted. Under no circumstances does omitting a study result in a significant deviation from the overall summary effect (RRR). In 2 instances, the RRR for Mediterranean: Asia becomes (just) non-significant (Parenta et al 1997 and Varas-Lorenzo et al 2019) 


\section{Supplementary $R$ code}

\# 1. Introduction ----

\# R script to perform analyses reported in:

\# Rabbiaatul Addawiyah Imawana, Daniel Robert Smith \& Michaela Louise Goodson (2020)

\# The relationship between Inflammatory Bowel Disease and Helicobacter pylori across

\# East Asian, European and Mediterranean countries: a metaanalysis.

\# Annals of Gastroenterology.

\# R Code written by:

\# Daniel R Smith; daniel.smith6@newcastle.edu.my

\# Rabbiaatul A B Imawana; R.A.Binti-Imawana2@newcastle. ac.uk

\# May-24-2020

\# 2. Libraries ----

library(metafor) \# Performing meta-analysis

library(plyr) \# Manipulating data

library(ggplot2) \# Visualising results

library(multcomp) \# Performing contrasts

library(gridExtra) \# Enhancing plots

library(dplyr) \# Manipulating data

library(stringr) \# Character string manipulation

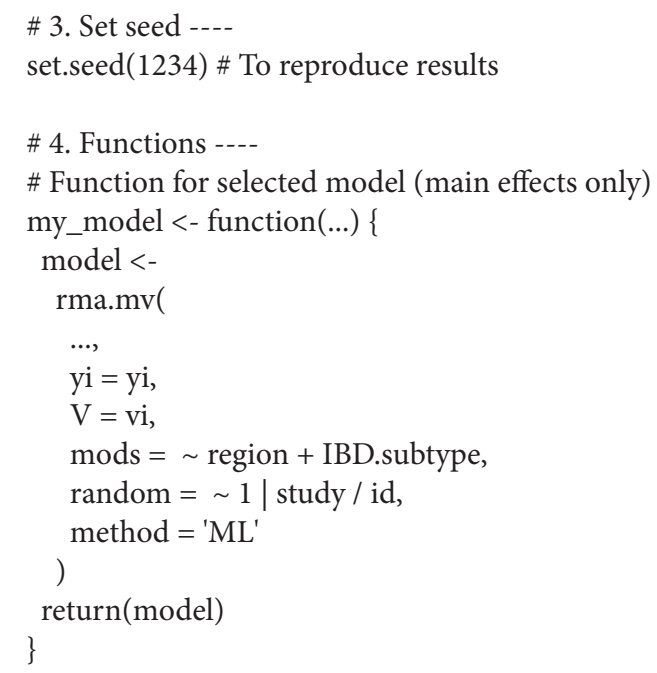

\# Function to compute relative risks and confidence intervals for each region $\mathrm{x}$ subtype combination

pred_fun_rr $<-$ function $(. .)\{$.

$\mathrm{p}<-\operatorname{predict}(\ldots, \operatorname{transf}=\exp , \operatorname{digits}=2)$

df1 <- data.frame $($ subtype.region = rownames $($ my_dummy_ matrix), p)

df 2 <- as.data.frame(str_split_fixed(df1\$subtype.region, "_", 2))

names $(\mathrm{df} 2)<-\mathrm{c}($ 'subtype', 'region')

df3 $<-$ cbind (df2, df1)

return(df3)

\}

\# Function to compute contrasts and confidence intervals (ratio of relative risks) cont_fun_rr $<-$ function $(. .)\{$.

cont $<-\operatorname{summary}(\operatorname{glht}(. .)$, test $=$ adjusted("single-step") $)$

conf $<$ - confint (cont)

pval $<-$ conf\$test\$pvalues

df1 <- data.frame $(\exp ($ conf\$confint $), p v a l=p v a l)$

$\mathrm{df} 2<-$ data.frame $($ contrast $=$ rownames $(\mathrm{df1}), \mathrm{df} 1)$

return(df2)

\}

\# Function to perform leave-one-out sensitivity analysis for predictions and contrasts

sen_fun $<$ - function(..., data, study_omit) \{

model $<-$ my_model $(\ldots$, data $=$ data[!data $\$ s t u d y==$ study_ omit, ])

preds1 <- pred_fun_rr(..., model, my_dummy_matrix)

preds $2<-$ data.frame(study $=$ study_omit, preds1)

$\mathrm{i}<-$ sapply(preds2, is.factor)

preds2[i] <- lapply(preds2[i], as.character)

cont $1<$ - cont_fun_rr(..., model, my_contrast_matrix)

cont $2<-$ data.frame(study = study_omit, cont 1 )

$\mathrm{j}<-$ sapply(cont2, is.factor)

cont2[j] <- lapply(cont2[j], as.character)

mylistout $<-$ list $($ preds $=$ preds 2 , contrasts $=$ cont 2$)$

return(mylistout)

\}

\# 5. Load \& format data ----

\# Read in data from master csv file

df $<-$ read.csv(file = "IBD_Hpylori_master.csv")

\# Tidy up study names

df\$study <-

as.factor(word(df\$name, 1, sep = "_"))

\# 6. Statistical analysis -----

\# Compute effeCt sizes (relative risks)

es $<$ -

summary(

escalc(

measure = "RR",

$\mathrm{ai}=$ Hppov.IBD,

bi $=$ Hpneg.IBD,

$\mathrm{ci}=$ Hppov.control,

$\mathrm{di}=$ Hpneg.control,

data $=\mathrm{df}$

)

)

\# add observation id column

es\$id <- 1:nrow(es)

\# fit full mixed effets model including interaction term $\bmod 1<-$

rma.mv(

yi $=y i$,

$\mathrm{V}=\mathrm{vi}$,

mods $=\sim$ region ${ }^{*}$ IBD.subtype,

random $=\sim 1 \mid$

study / id, 
data $=$ es,

method $=$ 'ML'

) \# includes interaction effect

\# fit reduced form model excluding interaction term (i.e. only main effects)

$\bmod 2<-$ update $(\bmod 1, \operatorname{mods}=\sim$ region + IBD.subtype $)$

\# perfom a liklihood ratio test of full and reduced models and print results

writeLines("Liklihood ratio test of mod1 (full) and mod2 (reduced) models.....")

print $(\operatorname{anova}(\bmod 1, \bmod 2))$

cat(' ', sep="\n\n")

\# print results for mod2 (selected model for paper)

writeLines("Model used in paper.....")

$\operatorname{print}(\bmod 2)$

cat(' ', sep="\n\n")

\# perform Wald test for region

writeLines("Wald test for region...")

$\operatorname{print}(\operatorname{anova}(\bmod 2, \mathrm{btt}=2: 3))$

cat (' ', sep="\n\n")

\# perform wald test for subtype

writeLines("Wald test for subtype...")

$\operatorname{print}(\operatorname{anova}(\bmod 2, \mathrm{btt}=4))$

cat(' ', sep="\n\n")

\# Compute generalized $\mathrm{I}^{\wedge} 2$

\# Formulae obtained from: http://www.metafor-project.org/ doku.php/tips:i2_multilevel_multivariate

$\mathrm{W}<-\operatorname{diag}(1 /$ es\$vi)

$\mathrm{X}<-$ model.matrix $(\bmod 2)$

$\mathrm{P}<-\mathrm{W}-\mathrm{W} \%{ }^{\star} \% \mathrm{X} \%{ }^{\star} \%$ solve $\left(\mathrm{t}(\mathrm{X}) \%{ }^{*} \% \mathrm{~W} \% \%^{\star} \% \mathrm{X}\right) \%{ }^{\star} \% \mathrm{t}(\mathrm{X})$ $\%$ \% W

writeLines("Overall I^2=....")

$\operatorname{print}(100 * \operatorname{sum}(\bmod 2 \$ \operatorname{sigma} 2) /(\operatorname{sum}(\bmod 2 \$ \operatorname{sigma} 2)+$ $(\bmod 2 \$ \mathrm{k}-\bmod 2 \$ \mathrm{p}) / \operatorname{sum}(\operatorname{diag}(\mathrm{P}))))$

cat(' ', sep="\n\n")

\# Define dummy matrices for predictions and contrasts my_contrast_matrix $<-$

rbind (

'UC:CD' $=c(0,0,0,1)$,

'Europe:Asia' $=\mathrm{c}(0,1,0,0)$,

'Mediterranean:Asia' $=c(0,0,1,0)$,

'Mediterranean:Europe' $=c(0,-1,1,0)$

)

my_dummy_matrix $<-$

rbind

"CD_E. AS" $=\mathrm{c}(0,0,0)$,

"CD_EUR" $=c(1,0,0)$,

"CD_MED" $=\mathrm{c}(0,1,0)$,

"UC_E. AS" $=\mathrm{c}(0,0,1)$,

"UC_EUR" $=c(1,0,1)$,

"UC_MED" $=c(0,1,1)$

)
\# Compute and print relative risks for region $\mathrm{x}$ subtype combinations

df_rr_all_mods_comb <- pred_fun_rr(mod2, my_dummy_ matrix)

df_rr_all_mods_comb\$study <-

'All' \# 'All' required as indicator for sensitivity analysis that follows...

writeLines('Relative risk and confidence intervals by region and subtype.....')

print(df_rr_all_mods_comb)

cat(' ', sep="\n\n")

\# Compute and print contrasts (ratios of relative risks)

df_rrr_cont <-cont_fun_rr(mod2, my_contrast_matrix)

df_rrr_cont\$study $<-$

'All' \# mark that used all studies so can include on leave one out plot below...

writeLines('Ratios of relative risks and confidence intervals')

print(df_rrr_cont)

cat(' ', sep="\n\n")

\# 7. Diagnostics ----

\# Sensitivity analysis (leave-one-out)

\# Define list of studies

ls_studies <- as.list(levels(es\$study))

\# Fit model to orginal data, iteratively omitting one study per loop. Store predictions and contrasts in a list

sen_list <-

lapply(ls_studies, function(x)

sen_fun $($ data $=$ es, study_omit $=\mathrm{x}))$

\# Subset list for predictions and bind to make dataframe sen_df_preds <- bind_rows(lapply(sen_list, '[[', "preds"))

\# Subset list for contrasts and bind to make dataframe sen_df_contrasts <- bind_rows(lapply(sen_list, '[[', "contrasts"))

\# Create dataframe for predictions plot by binding original and leave one out analyses

sen_df_preds_plot <- rbind(sen_df_preds, df_rr_all_mods_ comb)

sen_df_preds_plot\$study $\quad<-\quad$ as.factor(sen_df_preds_ plot\$study)

\# Create dataframe for contrasts plot by binding original and leave one out analyses

sen_df_contrasts_plot <-rbind(sen_df_contrasts, df_rrr_cont)

sen_df_contrasts_plot\$study <- as.factor(sen_df_contrasts_ plot\$study)

\# Pseudo egger test for small-study bias

\# Note the addition of study variances (vi) to the model

mod2_egg <- update $(\bmod 2, \bmod s=\sim$ vi + region + IBD. subtype)

writeLines("Pseudo Egger-test for reduced model (see P value corresponding to intercept).....")

print(mod2_egg)

cat(' ', sep="\n\n") 


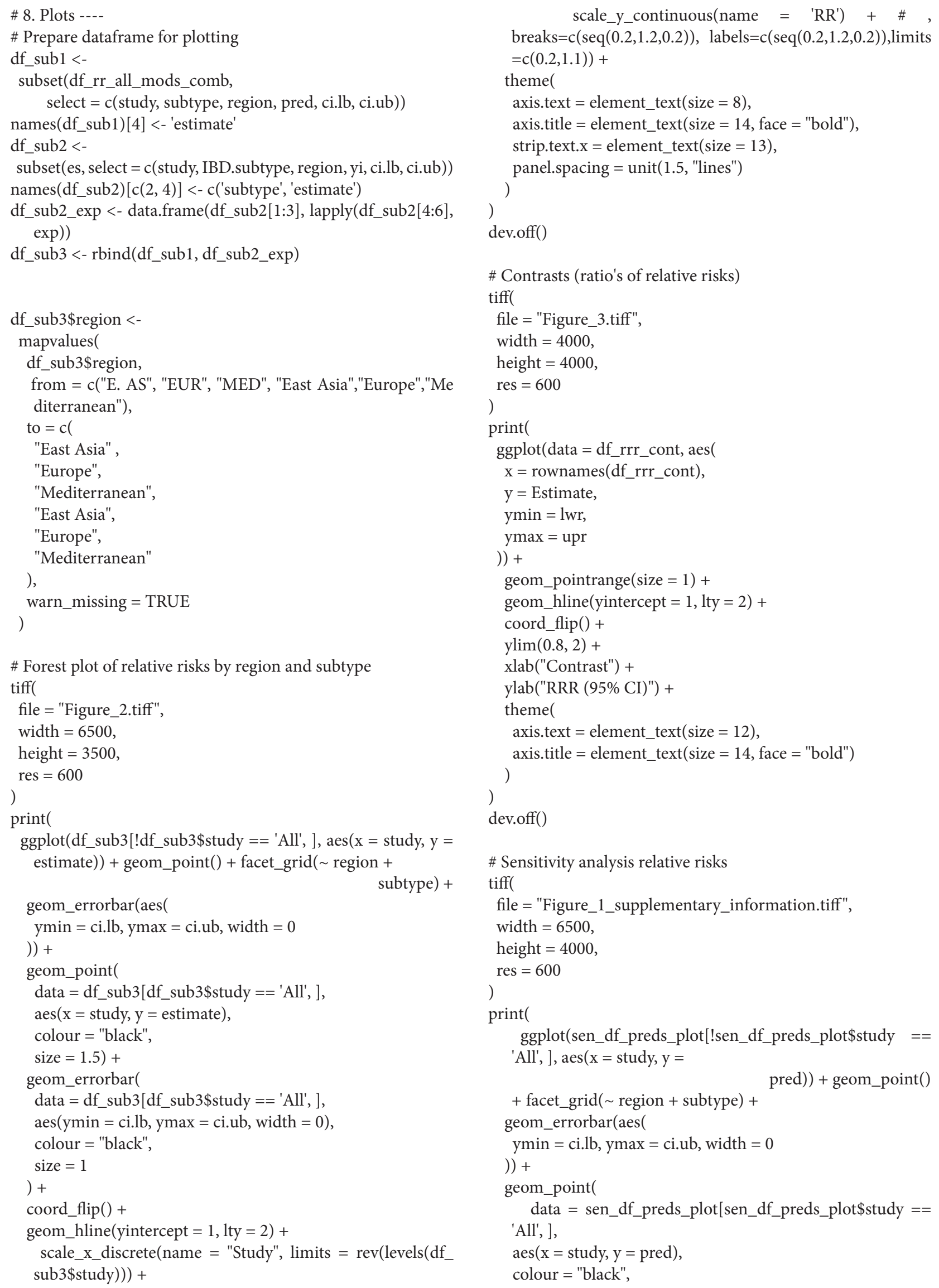




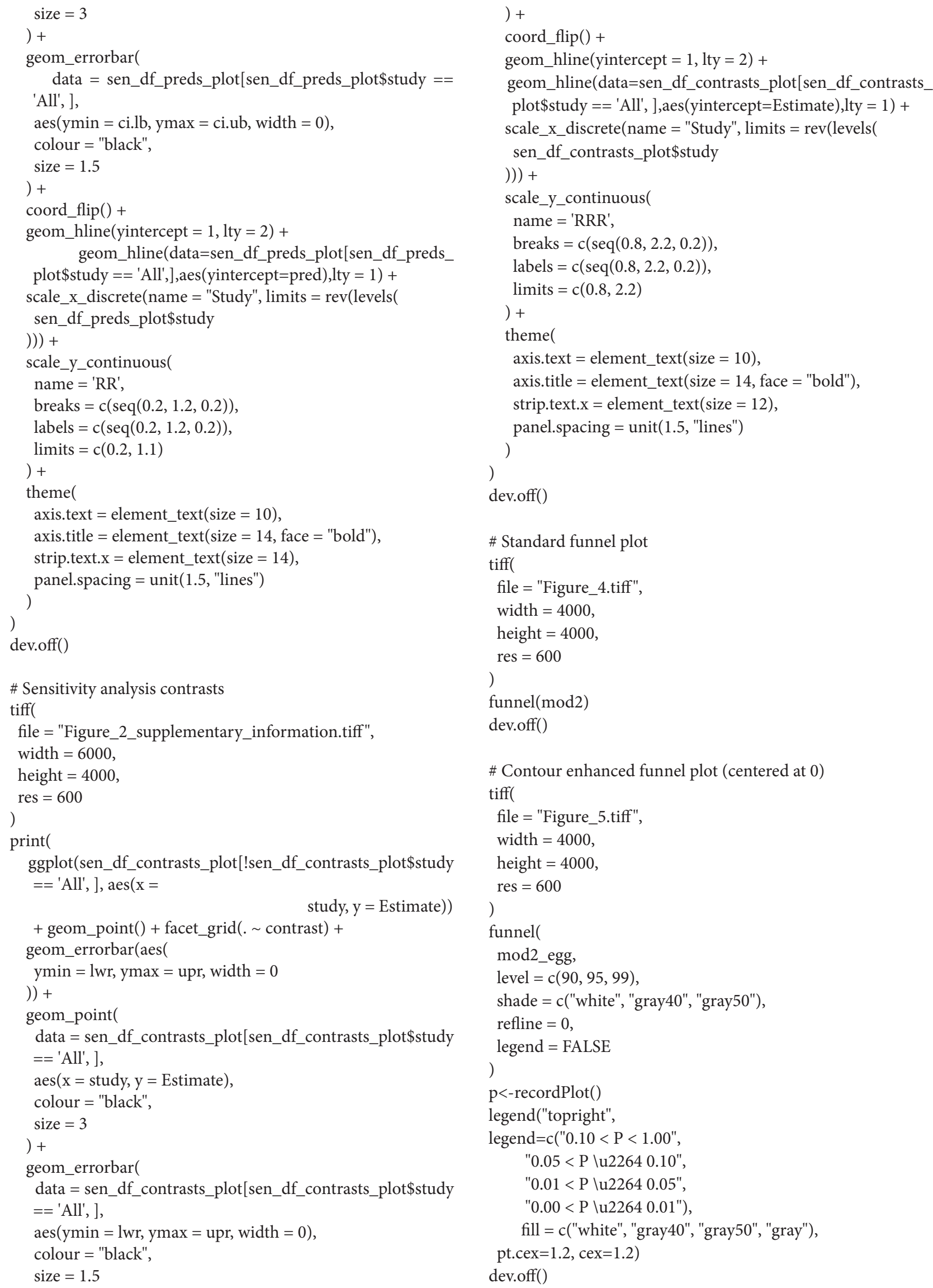

